\title{
Effects of part build orientations on fatigue behaviour of FDM-processed PLA material
}

\author{
Mst Faujiya Afrose $^{1} \cdot$ S. H. Masood ${ }^{1} \cdot$ Pio Iovenitti $^{1} \cdot$ Mostafa Nikzad $^{1}$. \\ Igor Sbarski ${ }^{1}$
}

Received: 1 June 2015/Accepted: 19 October 2015/Published online: 10 November 2015

(C) Springer International Publishing Switzerland 2015

\begin{abstract}
This paper investigates the fatigue behaviour of polylactic acid (PLA) parts processed by fused deposition modelling (FDM) additive manufacturing process. PLA is becoming a commonly used thermoplastic in open-source FDM machines for various engineering applications and it is essential that mechanical properties and performance of FDM-processed PLA parts must be properly understood. Very little data exist on the fatigue performance of PLA parts processed by FDM additive manufacturing. This study looks at the effect of part build orientations on the tensile fatigue properties of PLA material. A Cube 3D printer was used to print dog-bone test specimens in three $\left(\mathrm{X}, \mathrm{Y}\right.$ and $45^{\circ}$ ) different build orientations. These dog-bone parts were based on ASTM D638 standard and were cyclically tested at 80,70,60 and 50\% nominal values of the ultimate tensile stress by using a Zwick Z010 universal testing machine. Results show that in static loading, the FDM-processed PLA parts in X build orientation exhibit higher tensile stress, in the range of $60-64 \%$ of that of injection moulded PLA material, compared to those built in $\mathrm{Y}$ and $45^{\circ}$ orientations. But under tensile cyclic loading
\end{abstract}

\section{S. H. Masood \\ smasood@swin.edu.au \\ Mst Faujiya Afrose \\ mafrose@swin.edu.au \\ Pio Iovenitti \\ piovenitti@swin.edu.au \\ Mostafa Nikzad \\ mnikzad@swin.edu.au \\ Igor Sbarski \\ isbarski@swin.edu.au}

1 School of Engineering, Swinburne University of Technology, Hawthorn, VIC 3122, Australia condition, the parts in $45^{\circ}$ build orientation show higher fatigue life than the parts in $\mathrm{X}$ and $\mathrm{Y}$ build orientations for the same percentage of applied static loads. This paper adds knowledge to fill the gap on the fatigue characteristics of the PLA parts processed through FDM and would be useful in engineering design applications of such parts subjected to cyclic loading conditions.

Keywords Fused deposition modelling .

Thermoplastics · Polylactic acid · Tensile fatigue

\section{Introduction}

Additive Manufacturing (AM) is a novel technology that enables rapid fabrication of physical models directly from three-dimensional computer-aided design (CAD) data without any conventional tooling or programming requirements. It offers greater design flexibility and allows companies to turn first and effective design ideas into successful prototypes and end products rapidly and efficiently. First additive manufacturing systems appeared in 1986 with the introduction of Stereolithography technology [1]. In early 1990s, other technologies were commercialised including fused deposition modelling (FDM), laminated object manufacturing (LOM) and selective laser sintering (SLS). Stratasys introduced fused deposition modelling (FDM) technology in 1991, which has since become the most widely and commonly used AM process, which builds parts in a layer-by-layer manner by extruding semi-molten thermoplastic materials through a liquefier nozzle on to a platform [2]. Recently, several entry level and open-source FDM type machines have appeared in the market, which can process a variety of thermoplastic materials. These low cost AM machines not only serve as a 
means of learning and communications in education [3] but also offer applications in design verification and functional testing of engineering parts. The Cube 2 3D Printers are low cost small machines offering faster printability with acrylonitrile butadiene styrene (ABS) and polylactic acid (PLA) materials [4].

Because of light weight, ease of fabrication of complex geometry and low cost, such thermoplastics parts processed by FDM have been applied at a significant pace in engineering and medical fields. Though the load-bearing parts in industry are common engineering applications of thermoplastics [5], the leading applications of FDM thermoplastics are in biomedical and tissue engineering fields such as novel scaffold architectures [6] and knotless suture anchor [7]. Thus the knowledge of mechanical properties of FDM materials is significant now-a-days. Many studies have been done on ABS and polycarbonate (PC) thermoplastics to evaluate their mechanical properties as well as fatigue data. Lee and Huang [8] have investigated the fatigue data for several print orientations of ABS and ABSplus materials processed by Stratasys FDM using a Tinius Olsen H50KS testing machine. Also Ziemian et al. [9] have investigated different build orientations of ABS material to improve the mechanical properties. Masood et al. [10] have determined the tensile strength of FDMprocessed polycarbonate (PC) and found it to be in the range $70-75 \%$ of the moulded and extruded $\mathrm{PC}$ parts. Alhubail et al. [11] have optimised the FDM process parameter of ABS-M30i biomedical material by using Taguchi method and determined the surface roughness and tensile strength which results in improved part quality.

It is well known that mechanical properties including fatigue properties of plastic materials are affected by the type of manufacturing processes used in making plastic products. It is also known that layer-by-layer additive process impart anisotropic properties in the built parts and the AM process parameters also affect material properties of the processed material. This is especially true for FDM type process, which offers a wide range of process parameters to influence the mechanical properties of the FDM-processed parts. It is, therefore, necessary to understand how such properties of different plastics processed by various FDM processes vary due to different process parameters and build orientations. There have been some studies of mechanical properties of well-known additive manufacturing materials such as ABS, but other 3D printing materials such as PLA have received very little attention. In the published literature, some researchers have investigated the mechanical properties of PLA as composite materials added with many fibres [12, 13]. Jamshidian et al. [14] have presented a comprehensive review of PLA properties and modifications via different methods, like using modifiers, blending, copolymerizing, and physical treatments, and have also discussed industrial processing methods for producing different PLA films, wrappings, laminates, containers, bottles and cups). Tymark et al. [15] have recently investigated the mechanical properties such as tensile strength and modulus of elasticity of PLA materials processed by RepRap 3D printers.

It is noted that fatigue properties of FDM-processed PLA has not yet been addressed by researchers in published work. Polylactic acid (PLA) is a compostable, biodegradable thermoplastic made from renewable sources. PLA's good appearance, high mechanical strength, low toxicity; and good barrier properties have contributed in expanding its applications where the parts may encounter cyclic loading conditions. Therefore, in this paper, an experimental work is presented to investigate the fatigue behaviour of FDM-processed PLA materials through using flat dog-bone specimens under tensile cyclic loading. A universal testing machine was used to conduct fatigue testing at applied loads of different percentages of ultimate tensile strength (UTS). The effect of build orientation was investigated to understand the fatigue characteristics of the PLA parts to obtain data helpful in design of such parts subjected to cyclic loading conditions.

\section{Part fabrication using FDM}

The FDM type process used in the Cube-2 3D printer was employed to fabricate the dog-bone shaped tensile samples in $\mathrm{X}-, \mathrm{Y}$ - and $45^{\circ}$ - build orientations. The Cube 3D printers are based on FDM type plastic jet printing technology supplied by 3D Systems Inc. [16]. The filament material cartridges that work with the Cube come in different colours and can print around 13-15 medium size models from a single cartridge. The Cube does not require any support when part features are not angled more than $45^{\circ}$ in the print platform. Also, it allows moving the print head and the platform together in $\mathrm{X}, \mathrm{Y}$ and $\mathrm{Z}$ directions. In order to build a part, Cubify software converts the 3D STL files into printer cube files and offers three different print modes called Solid, Strong and Hollow. Solid mode is suitable for making solid functional model with best structural integrity. Hollow mode provides thin roads with air gaps and suitable for faster build. Strong mode is a mid-way between Solid and Hollow modes using moderate road widths and air gaps.

The print head heats the thermoplastics to molten state and produces a thin flowing material of plastic creating $0.20 \mathrm{~mm}$ thickness of layers that adheres to the print platform. The print head moves in one horizontal axis, while the table moves in the other horizontal and vertical directions. After each layer is produced, the print platform 


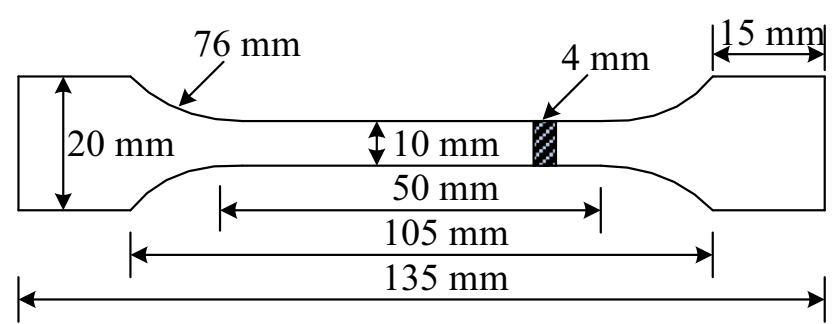

Fig. 1 Dimensions of the specimen

lowers so that a new layer can be drawn on top of the last. This process continues until the last layer on the top of the part is jetted.

In this study, the geometry of each fabricated dog-bone shape specimen was taken according to ASTM D638 to investigate the fatigue properties in tension [17]. Figure 1 shows the dimensions of the specimen used, which could be fitted on the Cube 3D Printer. The 3D CAD model was created using Creo parametric software and then converted into Stereolithiography (STL) file. To achieve desired creation, the chord height was set to 0 and angle control was set to 1 while saving as STL file for the Cube software.

Typically building a part using different print modes and different build directions will affect the part strength and mechanical properties. In this study, Solid print mode and three build orientations (X-, Y-, and $45^{\circ}$-) were used. Solid print mode provides zero air gaps between the depositing roads. Figure 2 shows the three build orientations in the Cube software to make the PLA samples. Forty samples of PLA materials in different orientations were printed to carry out several tensile and fatigue testing. Since Cube print head always moves parallel to the X-direction, the deposited road pattern direction in the part will be affected by the build placement of the part on the build table. Figure 3 shows the deposited material tool path road pattern used in the tensile specimen for each of the three build orientations.

\section{Fatigue testing}

Fatigue tests are considered when parts are expected to perform under cyclic load applications. In recent years, researchers have paid more attention on the fatigue behaviours of plastics as plastics are increasingly being used in aerospace, automotive, biomedical and other leading industries. Like all engineering materials, if plastic parts are considered under repetitive loading then it is important to know the fatigue life of such parts. In general, thermoplastics are more sensitive to various parameters and these parameters include stress or strain amplitude of the loading cycle, mean stress, stress or strain rate, initial defects present in the component, temperature, frequency and environment. These factors are to be considered when designing the part for the fatigue life under cyclic loading and would provide a better understanding to define materials to be used in specific applications.

For fatigue testing, a Zwick Z010 universal testing machine was used, which allows a maximum $10 \mathrm{kN}$ load capacity. The machine was controlled by testXpert ${ }^{\circledR}$ II intelligent software to control and record all test data. It was observed that a higher frequency increases the body temperature of the specimen, which results in the decrease of fatigue life by enabling material flow and increasing ductility, localised deformation at the weakest section of the gauge length. Conversely, a lower frequency results in an increased fatigue life appearing mostly in brittle fracture with limited deformation over the gauge length [18]. So the tests were set at a frequency of $1 \mathrm{~Hz}$ at room temperature. No specimen temperature control device was supplied during the test due to the requirements defined for the test programme. The wedge style cross-hatched grips were used for proper griping of the specimens as shown in Fig. 4.

In order to do fatigue tests, it was important to know the ultimate tensile strength (UTS) of specimens, and therefore, three PLA specimens were tested statically to determine the UTS for each of the three distinct orientations at a strain rate of $50 \mathrm{~mm} / \mathrm{min}$ as reported earlier [19]. The static tensile testing of the PLA plastic specimen was conducted according to ASTM D638 using the same Zwick Z010 testing machine. Five samples for each build orientation were used with a single pull until failure to determine ultimate tensile stresses and to average the tensile results. Figure 5 shows the static stress-strain curve obtained for the PLA samples for the 3 build orientations. In cyclic test programme, the test parameters were kept unchanged for each tested specimens at various applied load conditions over the cycles. To set the number of cycles, three specimens for the three different orientations were tested and then set to 5000 cycles to overcome the data overflow in the test programme. The applied load was varied at 50, 60, 70 and $80 \%$ of UTS from specimen to specimen during testing. Due to time consuming nature of cyclic loading experiment in a tensile tester, only one specimen was tested for each orientation and maximum load as the objective was to see the trend in the fatigue behaviour of PLA parts in build orientations.

\section{Results and discussion}

As noted earlier, all samples were subjected to uniaxial tension while conducting static and fatigue tests. The pullout and retraction were controlled within its maximum and 
Fig. 2 Build orientations in Cube software

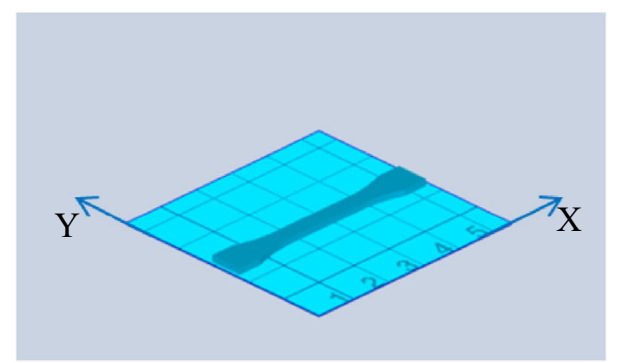

(a) X-orientation

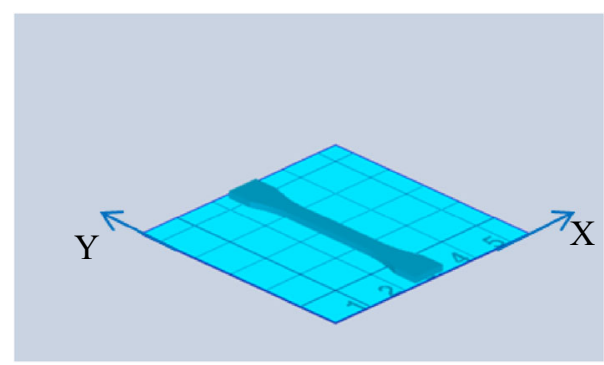

(b)Y-orientation

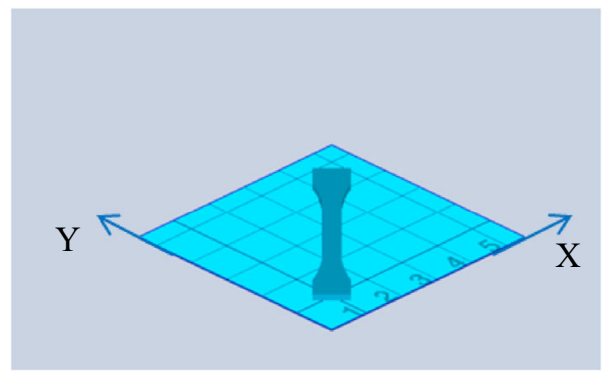

(c) $45^{\circ}$-orientation

Fig. 3 Deposited toolpath pattern on the specimen in three build orientations

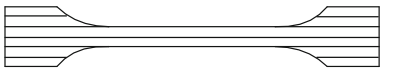

(a) X-orientation

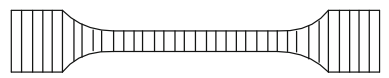

(b) Y-orientation

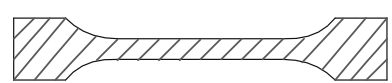

(c) $45^{\circ}$-orientation

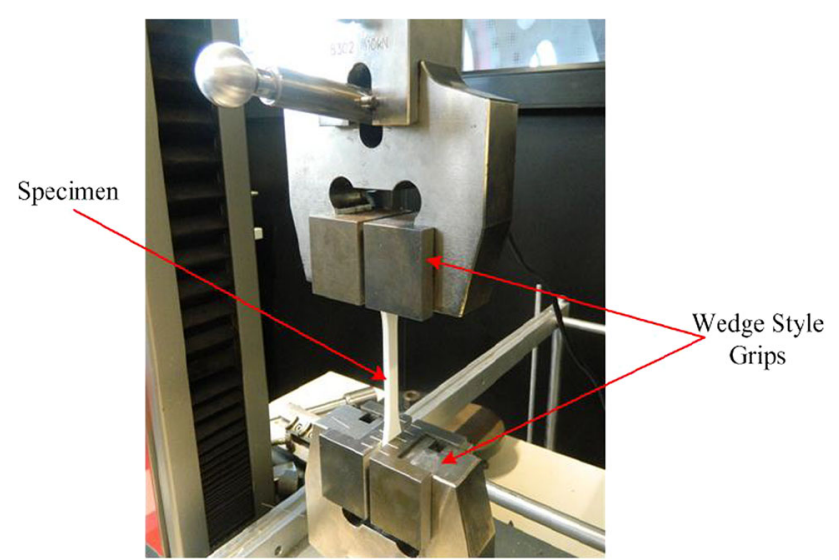

Fig. 4 Specimen holding in a $10 \mathrm{KN}$ Zwick machine

minimum load for each specimen. Static stress-stress graphs of Fig. 5 show that the ultimate tensile stresses of fabricated specimens for the three different orientations are different since it depended on build styles and orientations, rather than the material itself.

Though the tensile strength of the raw material was more consistent and higher than the FDM specimens, it was observed that the tensile stress was around $60-64 \%$ of the

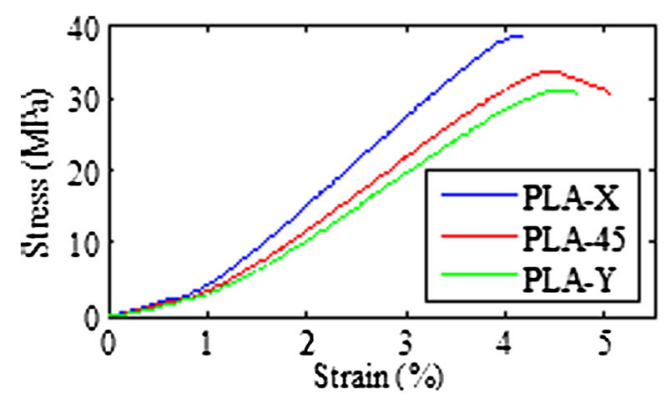

Fig. 5 Static stress vs. strain graph of PLA tensile specimens built in different orientations

generic raw PLA materials [19]. The output excel file of tensile test provides tables for maximum force, elongation at maximum force, force at break and elongation at break. The data were post-processed into stress, strain and number of cycles using Matlab script. Table 1 shows the average values of UTS and average values of modulus of elasticity obtained by static testing and the amount of applied stress at 80, 70, 60 and $50 \%$ of UTS used during fatigue testing for each of these three orientations. Figure 6 shows the typical pull history while cyclically loading at $50 \%$ of UTS for the specimens of three orientations named as PLA-X, PLA-Y and PLA-45, 
Table 1 Data outlining the average ultimate tensile stress $\left(\sigma_{\mathrm{u}}\right)$, average modulus of elasticity and applied load in percentage of UTS

\begin{tabular}{|c|c|c|c|c|c|c|}
\hline \multirow{2}{*}{$\begin{array}{l}\text { Orientation of } \\
\text { specimen }\end{array}$} & \multirow{2}{*}{$\begin{array}{l}\text { Ultimate tensile stress } \\
\text { (UTS) } \sigma_{\mathrm{u}}(\mathrm{MPa})\end{array}$} & \multirow{2}{*}{$\begin{array}{l}\text { Modulus of } \\
\text { elasticity (MPa) }\end{array}$} & \multicolumn{4}{|c|}{ Applied stress (\%UTS) (MPa) } \\
\hline & & & $80 \%$ & $70 \%$ & $60 \%$ & $50 \%$ \\
\hline PLA-X & 38.7 & 1538 & 30.96 & 27.09 & 23.22 & 19.35 \\
\hline PLA-Y & 31.1 & 1246 & 24.88 & 21.77 & 18.66 & 15.55 \\
\hline PLA-45 & 33.6 & 1350 & 26.88 & 23.52 & 20.16 & 16.8 \\
\hline
\end{tabular}

Fig. 6 PLA specimens showing the pull history at $50 \%$ of the ultimate tensile stress

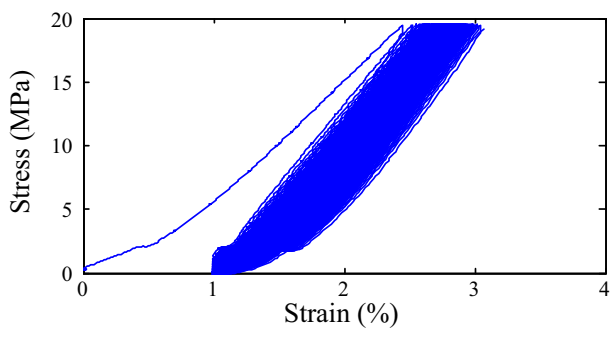

(a)X-orientation

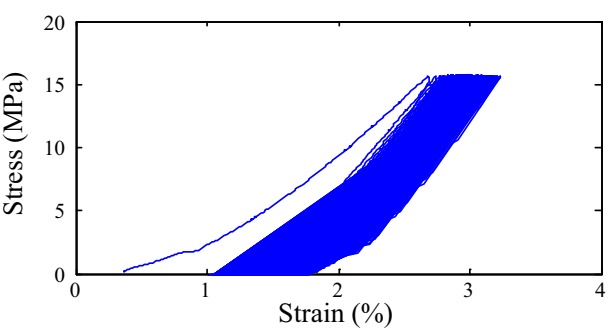

(b) Y-orientation

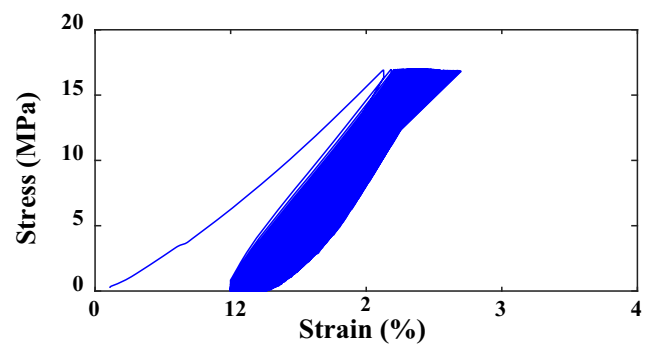

(c) $45^{\circ}$-orientation

respectively. It should be noted that stress-strain behaviour obtained under cyclic loading is quite different from that under static loading condition. In general, the yield strength in tension or compression gets reduced after applying cyclic load of the same magnitude but of opposite signs. The area within a hysteresis loop represents the energy dissipated during a cycle, usually in the form of heating. This energy represents the plastic work from the cycle. Depending upon the type of material used, the mechanism of cyclic hardening or cyclic softening also occurs due to dislocation of substructure within the material. Changes in cyclic deformation behaviour are more evident at the start of the cyclic loading and the material gradually stabilises with continued cycling. As shown in Fig. 6, PLA parts built in 45 orientation show higher cycle softening than the parts built in $\mathrm{X}$ - and Y-orientations.

As shown in Table 1, the specimen in Y-orientation has lower tensile stress than in $\mathrm{X}$ - and $45^{\circ}$-orientations. In $\mathrm{X}$-orientation, the deposited roads are aligned and bonded parallel to the longer dimension of the specimen, and therefore it offers maximum resistance to deformation in tension. In Y-orientation, the deposited roads are perpendicular to the longer dimension of the specimen (see Fig. 3), and therefore offer least resistance to deformation in tension. In 45 build orientation, the roads are inclined at 45 to longer length of specimen, and hence offer intermediate level of resistance to deformation. This trend is clearly visible in the measured values in Table 1 . However, as shown in Fig. 6, the Y-orientation has displayed better ductility as compared to other two orientations. Note that from stress-strain graphs, the modulus of elasticity of PLA for three distinct orientations were worked-out by plotting slope on its elastic region while specimens were tested to determine the UTS for each orientation.

Generally, parts fail in high stress concentration area under cyclic loading applications. For homogeneous parts, a failure should appear directly in the middle of the part. Figure 7 shows the failure profile of tested specimens for three build orientations. The failure profile of the specimens appears in different locations due to the different build style road pattern in each build orientations, which affect the material properties. From Fig. 7, it can be seen that the fatigue failure location for the $\mathrm{X}$-orientation specimens appeared consistently at the same location across the neck as 
Fig. 7 Overview of fatigue tested PLA specimens

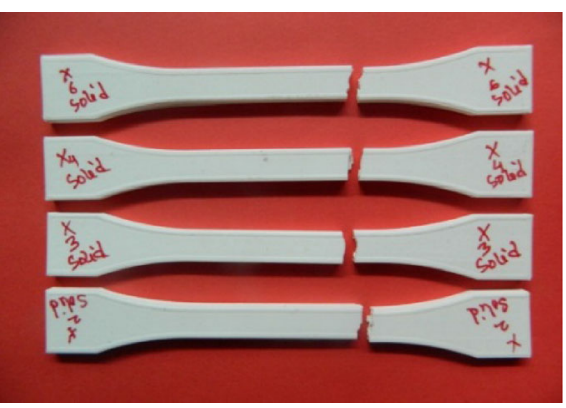

(a) X-orientation

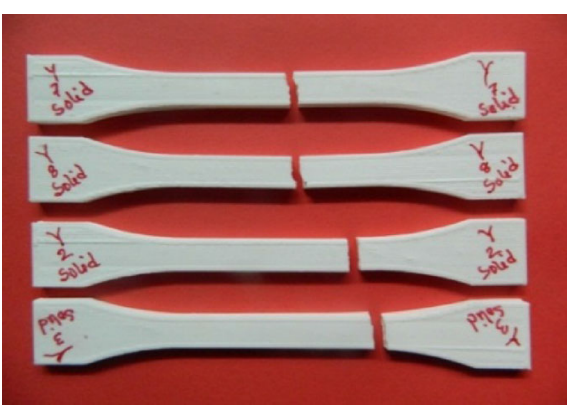

(b) Y-orientation specimens

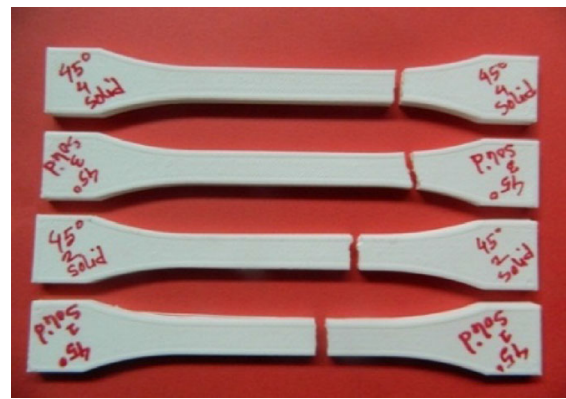

(c) $45^{\circ}$-orientation specimens

the build pattern roads are along the length of the tensile sample (see Fig. 4). In the Y-and $45^{\circ}$-orientation specimens, the build pattern roads were either perpendicular or at $45^{\circ}$ to the length of the sample, and this resulted in failure at different locations where the parts were highly stressed.

Because of the sensitivity in many factors, the fatigue test conditions must closely mimic the service conditions of the thermoplastic part and the $\mathrm{S}-\mathrm{N}$ approach is widely accepted in the engineering community for design applications when considering cyclic loading. Figure 8 shows the stress vs. numbers of cycles to failure curves ( $\mathrm{S}-\mathrm{N}$ curves) for $\mathrm{X}-, \mathrm{Y}-$ and $45^{\circ}$-orientations specimens subjected to static stress at their $50,60,70$ and $80 \%$ of UTS. Despite the inevitable scatter, the pattern of behaviour appears to be similar for all three build orientation parts and each point shows the failure point of each specimen when they are cyclically loaded at a certain percentage of their UTS value. Note that the average values of UTS for $\mathrm{X}$-, Y- and $45^{\circ}$ - orientation specimens were $38.7,31.1$ and 33.6 MPa, respectively, as in Table 1. From Fig. 8, it can be seen that although the X-orientation specimens experienced highest UTS, it generated lower fatigue life cycle than other two orientation specimens. However, the specimen in $45^{\circ}$-orientation had lower UTS than the X-orientation specimen, but it showed a higher number of fatigue life cycles than $\mathrm{X}$ - and $\mathrm{Y}$-orientation specimens. This trend is due to build orientations of printed specimens and build pattern road in relation to build direction. It was observed

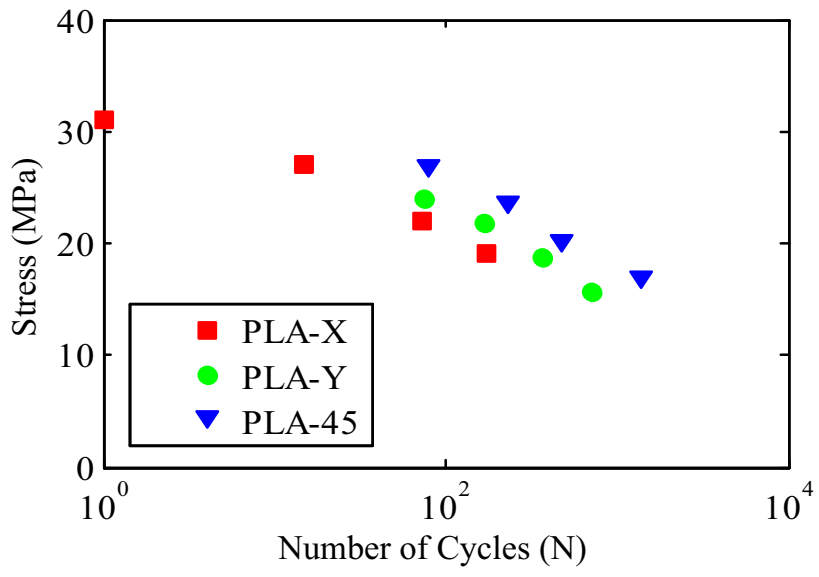

Fig. 8 S-N curves for three build orientations ( $\mathrm{X}, \mathrm{Y}$ and $45^{\circ}$ ) orientations

that for $45^{\circ}$-orientation specimen at approximately $50 \%$ of UTS, the number of cycles is roughly 1380 until its failure.The area under the stress-strain curve is the modulus of toughness or total strain energy per unit volume consumed by the material until failure. The strain energy can be calculated by using the following formula.

Total strain energy $(U)=\int_{0}^{\epsilon} \sigma d \epsilon$

where $\sigma$ is the stress and $\in$ is the strain. 
Therefore, if the stress-strain curve is integrated numerically, the total strain energy can easily be calculated. In this study, the total strain energy was calculated by using a Matlab function "trapz" which numerically calculates the total area under the stress-strain curve, i.e., the total strain energy. Figure 9 shows the total strain energies for three distinct build orientations for specimens stressed at $50 \%$ of their UTS. From Fig. 9, it can be observed that the $45^{\circ}$-orientation specimen experienced higher strain energy as compared to other build orientations with a value of $2048.9 \mathrm{~kJ} \mathrm{~m}^{-3}$ until it failed at 1380 cycles. On the other hand, the specimens in $\mathrm{X}$ - and $\mathrm{Y}$ - orientations experienced strain energy of 466.69 and $1421.69 \mathrm{~kJ} \mathrm{~m}^{-3}$, respectively, and the numbers of cycle until failure for Xand Y-orientations were 175 and 708, respectively. These three trends were consistently presented for all other tested specimens subjected to loading of 60,70 and $80 \%$ of UTS.

Typically the strain energy decreases while testing at higher tensile stress. Figure 10 shows the strain energy vs.

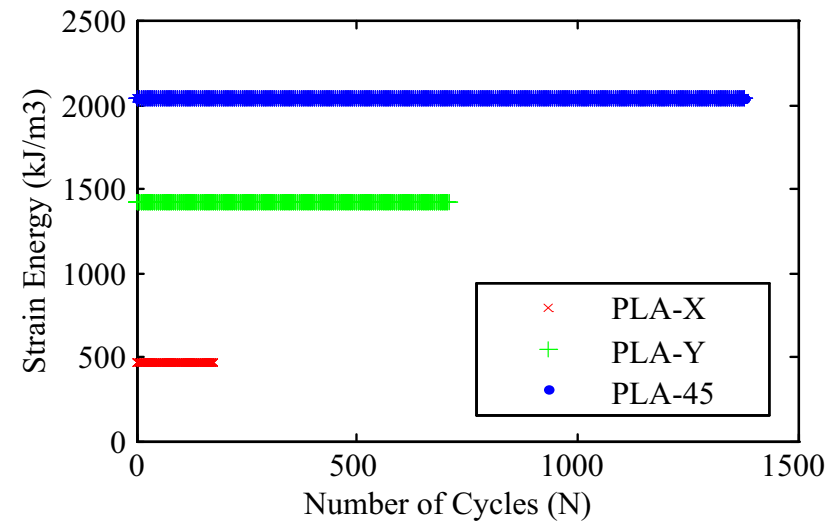

Fig. 9 Strain energy vs. number of cycles curves at $50 \%$ of UTS for the three build orientations

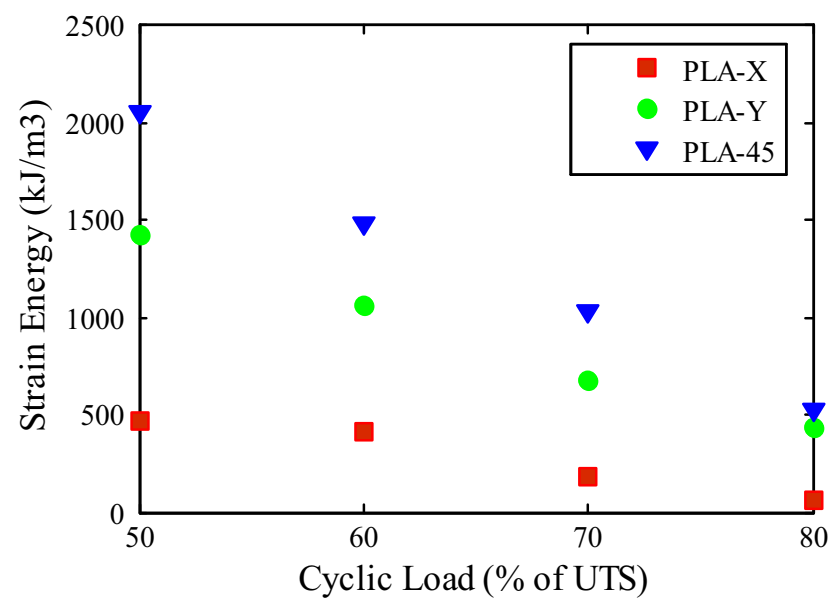

Fig. 10 Strain energy vs. percentage of UTS for specimens in different orientations (X, Y and $45^{\circ}$ ) cyclic load for $50,60,70$ and $80 \%$ of UTS for specimens in all three build orientations. From Fig. 10, it can be observed that the specimen in $45^{\circ}$-orientation experienced highest strain energy with respect to the percentage of cyclic loading conditions from other orientations. Thus, this study reveals that the PLA specimens printed in $45^{\circ}$-orientations have higher modulus of toughness, absorb more energy and last longer till failure under fatigue loading conditions compared to the PLA specimens built in the X- and Y-orientations specimens. This aspect is to be considered when designing FDM built parts for cyclic loading applications.

\section{Conclusions}

In this work, an experimental analysis of fatigue characteristics of fused deposition modelling processed PLA thermoplastics was carried out considering the effect of different build orientations. It was observed that the ultimate tensile stress of PLA samples built in X-direction (PLA-X) was found to be the highest at $38.7 \mathrm{MPa}$ and ranged from 60 to $64 \%$ of raw PLA material, while for PLA-Y and PLA-45, the values were lower at 31.1 and $33.6 \mathrm{MPa}$, respectively. Also, it was observed that under cyclic loading application the PLA specimens built in $45^{\circ}$ orientations achieved highest fatigue life compared to those PLA specimens built in X- and Y- orientations. PLA specimens built in 45-orientation also displayed best capacity to store strain energy compared to those built in other two orientations. This study will assist in developing design guidelines for application of FDM built parts used in cyclic loading conditions. Further work in this area will include an investigation on how the fatigue life and total strain energy may be influenced by different strain rates and frequency of tests. Moreover it will be useful to study the effect of temperature, environment, surface finish and mode of loading (compressive, flexural) to gather a more comprehensive knowledge on fatigue behaviour of FDM made parts. Such data would be extremely useful to design parts as more and more additive manufactured parts and materials are being applied to various engineering applications in different loading conditions.

Acknowledgments Authors acknowledge technical support of $\mathrm{Mr}$ Warren Gooch for specimens making in the product design lab.

\section{References}

1. Hull CW (1986) Apparatus for production of three-dimensional objects by Stereolithography. Patent 4, 575, 330, UVP, Inc., San Gabriel, CA

2. Chua CK, Leong KF, Lim CS (2003) Rapid prototyping: principals and applications, 2nd edn. World Scientific, Singapore 
3. Kostakis V, Niaros V, Giotitsas C (2014) Open source 3D printing as a means of learning: an educational experiment in two high schools in Greece. Telemat Inf 32:118-128. doi:10.1016/j. tele.2014.05.001

4. 3D Systems, Cube 2. http://www.3dsystems.com/zh/3d-printers/ personal/cube. Accessed 28 May 2015

5. Stokes VK (1995) Thermoplastics as engineering materials: the mechanics, materials, design, processing link. J Eng Mater Technol 117:448-455

6. Zein I, Hutmacher DW, Tan KC et al (2002) Fused deposition modeling of novel scaffold architectures for tissue engineering applications. Biomaterials 23:1169-1185. doi:10.1016/S01429612-(01)00232-0

7. Pietschmann MF, Froehlich V, Ficklscherer A et al (2008) Biomechanical testing of a new knotless suture anchor compared with established anchors for rotator cuff repair. J Shoulder Elb Surg 17:642-646. doi:10.1016/j.jse.2007.11.017

8. Lee J, Huang A (2013) Fatigue analysis of FDM materials. Rapid Prototyp J 19:291-299. doi:10.1108/13552541311323290

9. Ziemian C, Sharma M, Ziemian S (2012) Anisotropic mechanical properties of ABS parts fabricated by fused deposition modelling. In: Gokcek M (ed) Mechanical Engineering, InTech, pp 159-180

10. Masood SH, Mau K, Song WQ (2010) Tensile properties of processed FDM polycarbonate material. Mater Sci Forum 654-656:2556-2559

11. Alhubail M, Alenezi D, Aldousiri B (2013) Taguchi-based optimisation of process parameters of fused deposition modelling for improved part quality. Int J Eng Res Technol 2:2505-2519
12. Dong Y, Ghataura A, Takagi H et al (2014) Polylactic acid (PLA) bio composites reinforced with coir fibres: evaluation of mechanical performance and multifunctional properties. Compos Part A Appl Sci Manuf 63:76-84

13. Kasuga T, Ota Y, Nogami $M$ et al (2000) Preparation and mechanical properties of polylactic acid composites containing hydroxyapatite fibres. Biomaterials 22:19-23

14. Jamshidian M, Tehrany Muhammad Imran M, Jacquo M, Desobr S (2010) Poly-lactic acid: production, applications, nanocomposites, and release studies. Compr Rev Food Sci Food Saf 9:552-571

15. Tymark BM, Kreiger M, Pearce JM (2014) Mechanical properties of components fabricated with open-source 3-D printers under realistic environmental conditions. Mater Des 58:242-246

16. 3D Systems, Cube. http://www.3dsystems.com/. Accessed 28 May 2015

17. ASTM (2010) D638-10 Standard test method for tensile properties of plastics. American Society for Testing and Materials, USA

18. Zoroufi M (2013) Significance of fatigue testing parameters in plastics versus metals. Article in http://softlaunch.element.quba. co.uk/. Accessed 28 May 2015

19. Afrose MF, Masood SH, Nikzad M et al (2014) Effects of build orientations on tensile properties of PLA material processed by FDM. Adv Mater Res 1044-1045:31-34 